\title{
Key Genes and Genetic Interactions of Plant-Pathogen Functional Modules in Poplar Infected by Marssonina brunnea
}

\author{
Sisi Chen, ${ }^{1,2,3}$ Yanfeng Zhang, ${ }^{2,3}$ Yiyang Zhao, ${ }^{1,2,3}$ Weijie $\mathrm{Xu},{ }^{1,2,3}$ Yue $\mathrm{Li}^{1,2,3}$ \\ Jianbo Xie, ${ }^{1,2,3,+}$ and Deiqiang Zhang ${ }^{1,2,3,+}$ \\ ${ }^{1}$ Beijing Advanced Innovation Center for Tree Breeding by Molecular Design, College of Biological Sciences and Technology, \\ Beijing Forestry University, No. 35, Qinghua East Road, Beijing 100083, P. R. China \\ ${ }^{2}$ National Engineering Laboratory for Tree Breeding, College of Biological Sciences and Technology, Beijing Forestry \\ University, No. 35, Qinghua East Road, Beijing 100083, P. R. China \\ ${ }^{3}$ Key Laboratory of Genetics and Breeding in Forest Trees and Ornamental Plants, Ministry of Education, College of Biological \\ Sciences and Technology, Beijing Forestry University, No. 35, Qinghua East Road, Beijing 100083, P. R. China
}

Accepted 9 May 2020.

\begin{abstract}
Marssonina brunnea, the causative pathogen of Marssonina leaf spot of poplars (MLSP), devastates poplar plantations by forming black spots on leaves and defoliating trees. Although MLSP has been studied for over 30 years, the key genes that function during $M$. brunnea infection and their effects on plant growth are poorly understood. Here, we used multigene association studies to investigate the effects of key genes in the plant-pathogen interaction pathway, as revealed by transcriptome analysis, on photosynthesis and growth in a natural population of 435 Populus tomentosa individuals. By analyzing transcriptomic changes during three stages of infection, we detected 628 transcription factor genes among the 7,611 differentially expressed genes that might be associated with basal defense responses. Gene Ontology and Kyoto Encyclopedia of Genes and Genomes enrichment analyses revealed that transcriptomic changes across different stages of infection lead to the reprogramming of metabolic processes possibly related to defense activation. We identified 29,399 common singlenucleotide polymorphisms (SNPs) within 221 full-length genes in plant-pathogen interaction pathways that were significantly associated with photosynthetic and growth traits. We also detected 4,460 significant epistatic pairs associated with stomatal conductance, tree diameter, and tree height.
\end{abstract}

The raw sequence data are available in the Genome Sequence Archive in the Beijing Institute of Genomics BIG Data Center, Chinese Academy of Sciences under accession number CRA001647.

${ }^{\dagger}$ Corresponding authors: D. Zhang; DeqiangZhang@bjfu.edu.cn and J. Xie; jbxie@bjfu.edu.cn

Funding: This work was supported by the Open Project of Guangdong Provincial Key Laboratory of Silviculture, Protection and Utilization (SPU 2018-01), the Project of the National Natural Science Foundation of China (31600537 and 31972954), Young Elite Scientists Sponsorship Program by CAST (2018QNRC001).

*The $\boldsymbol{e}$-Xtra logo stands for "electronic extra" and indicates that supplementary figures and tables and supplementary supporting materials are published online.

The author(s) declare no conflict of interest.

(C) 2020 The American Phytopathological Society
Epistasis analysis uncovered significant interactions between 2,561 SNP-SNP pairs from different functional modules in the plant-pathogen interaction pathway, revealing possible genetic interactions. This analysis revealed many key genes that function during $M$. brunnea infection and their potential roles in mediating photosynthesis and plant growth, shedding light on genetic interactions between functional modules in the plant-pathogen interaction pathway.

Keywords: genetic interaction, Marssonina brunnea, photosynthesis and growth, plant-pathogen interaction, poplar, transcriptome

The long-term coevolution of plants and microbes has led to sophisticated invasion and defense mechanisms between the host and microbial pathogen, making plant-pathogen interaction networks quite complex (Chisholm et al. 2006). Plants are constantly exposed to microbial pathogens, which occupy the extracellular environment and attempt to obtain nutrients from host cells for their growth and reproduction (Dodds and Rathjen 2010). As pathogens have found ways to invade and communicate with their hosts, plants have evolved sophisticated immune systems to fight infection (Jin 2016). Whenever there is pathogen invasion, there is an increase in reactive oxygen species (ROS) production at the site of pathogen interaction. In pathogenesis, ROS provide the first line of defense and also serve as a secondary messenger to signal subsequent defense reactions in plants (Afzal et al. 2014). To ward off pathogens, plants induce innate immune responses that are often associated with a reduction in plant growth (Azevedo et al. 2018; Jones and Dangl 2006). This plant growth-defense balance is regulated by specific recognition and complex signaling transduction mechanisms. Especially in long-lived woody trees, plants use the mechanisms to convert resources for growth into defense-response pathways, activating appropriate defense responses through transcriptional reprogramming (Hamel et al. 2011; Pieterse et al. 2012).

Poplars (Populus spp.), comprising six taxonomic sections (Abaso Ecken, Turanga Bunge, Leucoides Spach, Aigeiros Duby, Tacamahaca Spach, and Leuce Duby), are important commercial tree species. Poplars are distributed worldwide and serve as a model woody perennial organism for researchers interested in testing mechanistic hypotheses related to 
plant-microbe interactions. Poplars are severely damaged by a variety of fungal diseases in nature, particularly when grown in plantations, which can dramatically limit productivity (Duplessis et al. 2009; Mutwil et al. 2011; Movahedi et al. 2012; Zhu et al. 2013). Marssonina leaf spot of poplars (MLSP) is a major disease of the genus Populus caused by members of the coelomycetous genus Marssonina Magnus (including Marssonina castagnei Magn., Marssonina populi Magn., Marssonina brunnea Magn., and Marssonina balsamiferae Hiratsuka.) (Han et al. 2000; Spiers 1984). MLSP is characterized by the formation of black spots on leaves, leading to a decline in photosynthetic efficiency and seriously affecting wood production and quality (Balasubramanian et al. 2009; Cheng et al. 2010; Zhang et al. 2007). M. brunnea, the most pathogenic species causing MLSP, is found in numerous regions of China and causes serious economic losses in poplar plantations (Spiers 1984). Additionally, M. brunnea strains have been classified into the flowing two specialized forms according to their host specificity and morphological characteristics, i.e., M. brunnea $\mathrm{f}$. sp. monogermtubi and $M$. brunnea $\mathrm{f}$. sp. multigermtubi (Li 1984). The former mainly attacks section Leuce of the Populus genus, while the latter is hosted by many species of the sections Aigeiros and Tacamahaca (Han et al. 2000).

Previous studies have detailed the infection process (Heather et al. 1975; Spiers 1984) and histopathologic characterization (Zhang et al. 2018a) of M. brunnea. At the molecular level, some secretory proteins including some potential effectors have been identified (Cheng et al. 2010) and some molecular marker technologies have been developed in M. brunnea (Han et al. 2000; Zhang et al. 2007, 2018b). The molecular processes underlying the poplar-Marssonina brunnea f. sp. monogermtubi interactions have remained blurry and require further examination. Elucidating the molecular mechanism of interaction between Marssonina brunnea f. sp. monogermtubi and Leuce will provide new insights into disease control of poplar. Transcriptome analysis has become a standard approach to identifying key poplar genes in disease response, and studies have applied this approach to poplar and M. brunnea (Chen et al. 2015; Ren et al. 2020; Zhang et al. 2018c). While most of these studies concentrated on the expression comparisons between a resistant and a susceptible variety, the genetic diversity of poplar resistance in $M$. brunnea might be underestimated using only these approaches.

Traditional molecular genetic analysis with transgenic methods in trees may potentially characterize the functions and interactions among genes. However, these approaches remain time-consuming and technically challenging in trees and may not provide a global view. With the advance in high-throughput genotyping technologies, genome-wide association or multisingle-nucleotide polymorphism (SNP) association approaches have emerged as a complementary tool for dissecting the interaction of complex genetic traits on plants, including disease resistance and abiotic stress tolerance (Du et al. 2018; Oreiro et al. 2020). Association genetics, in particular epistasis, can be used to define genetic interactions of genes in the same pathways (Roguev et al. 2008).

In this study, we performed a comprehensive timecourse investigation of the transcriptomes of poplar during three essential stages of infection with M. brunnea. We uncovered many key genes in nine functional modules of the plantpathogen pathway that were significantly differentially expressed, providing important insights into the dynamics of transcriptomic reprogramming during plant defense responses. To elucidate the potential functions of these key genes in balancing plant growth and defense and genetic interactions between the functional modules in the plant-pathogen pathway, we used SNP association and epistasis analyses to investigate the nature of the genetic variation of these genes and functional modules. The results provide important insights into the effects of genetic interactions on plant defense pathways. To our knowledge, this is the first time to combine transcriptome and association genetics to elucidate the interaction between poplar and M. brunnea.

\section{RESULTS}

\section{Microscopic and biochemical characterization of infected poplar.}

To elucidate the mode of infection and the mechanism of resistance of Populus in response to M. brunnea, we inoculated Populus tomentosa cv. LM50 clones with a Marssonina brunnea f. sp. monogermtubi conidial suspension and performed the microstructural analysis at different timepoints (12, 24, and 48 h) after inoculation (hpi) (Fig. 1A). At $12 \mathrm{hpi}$, the conidia germinated a germ tube and the tip of the germ tube expanded to form appressoria. At $24 \mathrm{hpi}$, infective vesicles had developed and proliferated in the host cells. At $48 \mathrm{hpi}$, intercellular infective hyphae formed within the host cells and the pathogens were in the biotrophic phase (Fig. 1A).

We also analyzed the samples biochemically, including analysis of superoxide dismutase (SOD), peroxidase (POD), and catalase (CAT) activity as well as proline (PRO) and malondialdehyde (MDA) contents. Nearly all these parameters changed significantly in response to infection. For example, SOD activity and PRO content increased significantly at $12 \mathrm{hpi}$ (Fig. 1B) $\left(P<1 \times 10^{-2}\right)$, while CAT activity and MDA content increased significantly at 48 hpi (Fig. 1B) $\left(P<1 \times 10^{-2}\right)$, compared with those in the control group (CK), which was sprayed with sterile distilled water and harvested at $12 \mathrm{hpi}$, suggesting that these antioxidant enzymes and the content of PRO and MDA might have different molecular bases for the corresponding mechanisms of expression regulation. Moreover, SOD activity reached the highest peak at $24 \mathrm{hpi}$ and increased by $77.73 \%$ compared with the CK (Fig. 1B). In total, the changes of antioxidant enzyme activities (SOD, POD, and CAT) and the content of PRO and MDA in poplar (P. tomentosa) leaf tissue after attack by $M$. brunnea indicate an early event of plant defense response in preventing oxidative damage caused by ROS.

\section{Transcriptomic responses to infection.}

We performed RNA-seq during the three major infection periods (covering the major transitions) of $P$. tomentosa leaves infected by M. brunnea. In total, $78.61 \mathrm{~GB}$ of clean data were obtained from the 12 samples $(12,24$, and 48 hpi and CK, three biological repeats) (Supplementary Table S1). The clean data of each sample exceeded 6.01 GB. The reads of an average quality score were greater than 30 (error rate 0.001 ), and the value of all samples exceeded $91.82 \%$. The proportion of mapped reads across all samples to the reference genome of $P$. trichocarpa ranged from 71.68 to $73.39 \%$ (Supplementary Table S1). The proportion of high-quality reads and mapping rates indicated that the RNA-seq data were of high quality and sufficient for subsequent functional annotation and other bioinformatics analyses. We conducted a pairwise differential analysis of gene-expression levels among the three stages of infection and used a $P$ value $\leq 5 \times 10^{-2}$ and $\log _{2}$ fold change $\left(\log _{2} \mathrm{FC} \mid \geq 1\right)$ as thresholds to retrieve the differentially expressed genes (DEGs).

We detected 6,034 DEGs (2,882 upregulated, 3,152 downregulated) between the $\mathrm{CK}$ and $12 \mathrm{hpi}, 2,769$ DEGs $(1,277$ upregulated, 1,492 downregulated) between 12 and $24 \mathrm{hpi}$, and 890 DEGs (335 upregulated, 555 downregulated) between 24 and 48 hpi (Fig. 2A). These results were confirmed by real-time quantitative PCR (qPCR) of nine genes detected by RNA-seq 
(Supplementary Fig. S1), despite differences in magnitude, indicating that the RNA-seq data are reliable. Notably, only three common upregulated DEGs (Potri.009G69100, Potri. 015G108500, and Potri.005G078700) and eight common downregulated DEGs (Potri.019G050200, Potri.017G012100, Potri.001G219300, Potri.015G000500, Potri.001G403300, Potri.013G121400, Potri.016G119100, and Potri.003G148900) were detected across all three stages of infection (Fig. 2B), suggesting that substantial transcriptome reprogramming occurs upon infection.

\section{Dynamic gene transcriptional profile \\ in response to $M$. brunnea.}

We performed $K$-means clustering analysis of the 7,611 DEGs described above $\left(P<5 \times 10^{-2}, \log _{2} \mathrm{FC}>1\right.$, removed duplications) (Supplementary Data S2) using the MultiExperiment Viewer package with $K$ set to 20. The clusters displayed highly dynamic gene expression patterns that might be associated with their functions in response to infection (Fig. 3A). Among these, the expression levels of 2,290 genes in cluster 16 and 17 showed a sustained decrease, whereas the expression levels of 656 genes in clusters 4 and 9 showed a sustained increase from -1 to 1 , suggesting that they are likely to play essential roles in plant responses to infection. Moreover, 1,284 genes in clusters 1, 3, 8, and 10 and 333 genes in clusters 6 and 7 were highly expressed at 12 and 24 hpi, respectively, suggesting that they also function in the early stages of infection (Fig. 3A).

Interestingly, we detected 628 transcription factor (TF) genes among the 20 clusters described above. MYB TFs participate in various processes in plants, such as responses to abiotic and biotic stress and defense responses (Kutter et al. 2012; Yanhui et al. 2006). Of these genes, genes encoding 27 MYB TFs (including MYB3, MYB4, MYB5, MYB12, and MYB94) were highly expressed, reflecting the important roles of MYB TFs in plant responses to infection. Also, the expression levels of genes encoding four important plant immune response-related WRKY TFs (WRKY11, WRKY18, WRKY33, and WRKY40), two AP2/ERF (ethylene-responsive factor) TFs (AP2.61 and AP2.12), four bZIP TFs (bZIP1, bZIP6, bZIP44, and bZIP53), five growth-regulating factor (GRF) TFs (GRF1, GRF2, GRF5, GRF7, and GRF9), and two ARF TFs (ARF8 and ARF11) uniformly increased throughout infection. Moreover, three MYBrelated TF genes (LHY1.1, LHY1.2, and Potri.016G83900; homeodomain-like superfamily protein) were highly expressed only at $12 \mathrm{hpi}$; these genes are associated with basal defense responses (Chen et al. 2018a).

\section{Transcriptional and functional enrichment analyses across different stages.}

To explore the biological functions of infection-responsive genes, we performed functional enrichment analysis to identify overexpressed gene ontology (GO) terms (Supplementary Data S2) $\left(P<1 \times 10^{-2}\right)$. Among the genes upregulated at $\mathrm{CK}$ versus $12 \mathrm{hpi}$, we detected an over-representation of genes involved in cell-wall activity (cell-wall organization or biogenesis, cellwall modification), metabolism (primary metabolic process, carbohydrate metabolic process, phosphate metabolic process, chitin metabolic process), and response to a biotic stimulus. However, we detected different functional profiles at 12 versus $24 \mathrm{hpi}$ and 24 versus 48 hpi. For example, upregulated genes involved in oxidation-reduction, carbohydrate metabolic
A

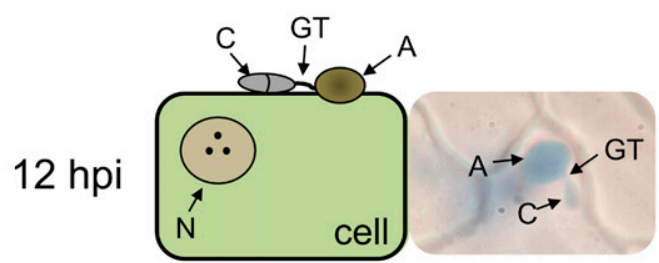

Spore germination
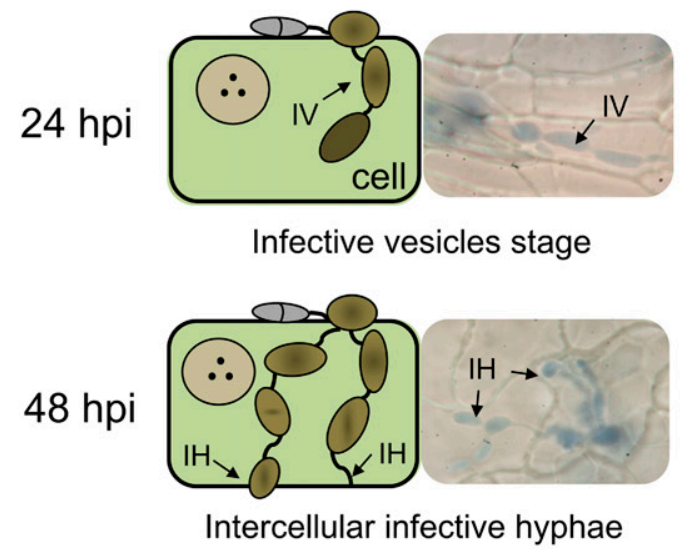
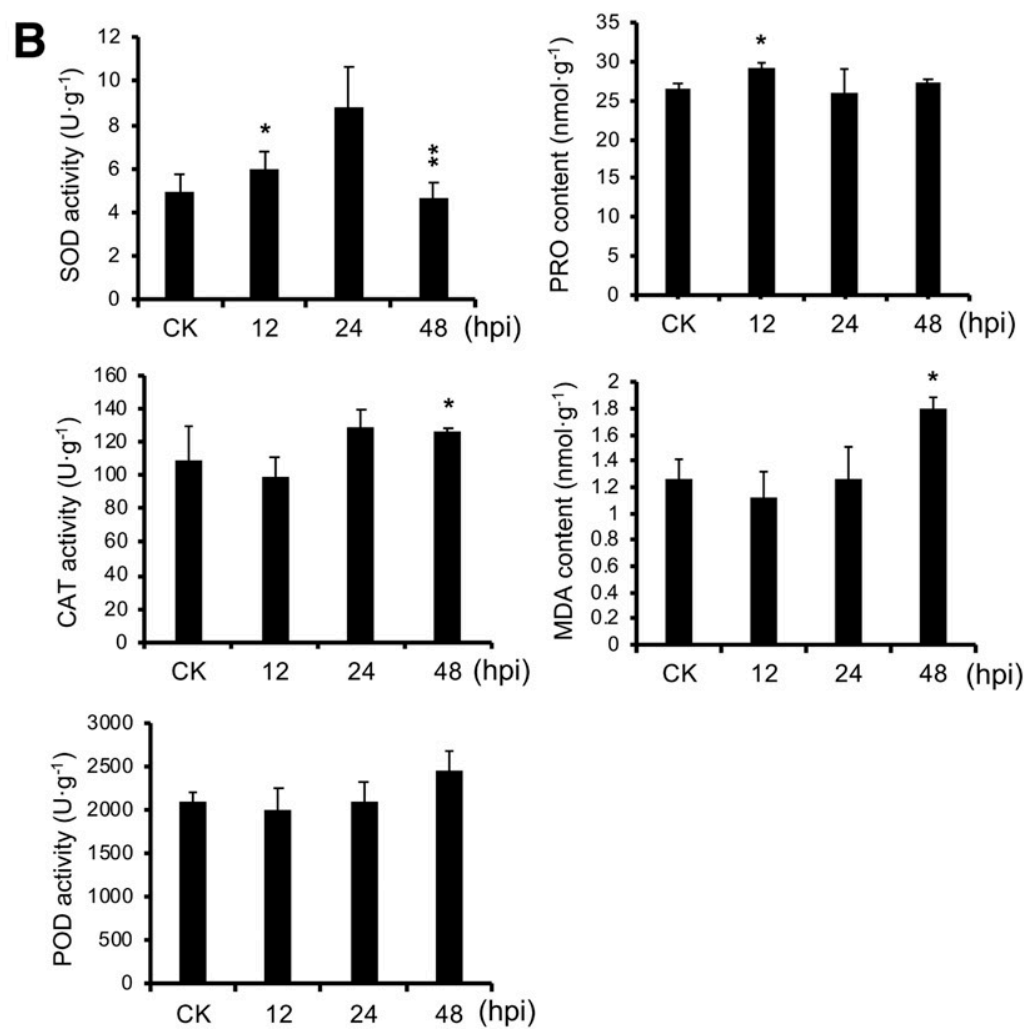

Fig. 1. Microstructural and biochemical characteristics during infection. A, Microstructures of inoculated poplar leaves at 12,24 , and 48 h postinoculation (hpi). $\mathrm{C}=$ conidia, $\mathrm{GT}=$ germ tube, $\mathrm{A}=$ appressoria, $\mathrm{IV}=$ infective vesicles, $\mathrm{IH}=$ intercellular infective hyphae, and $\mathrm{N}=$ nucleus. $\mathbf{B}, \mathrm{Biochemical}$ characteristics (superoxide dismutase [SOD], catalase [CAT], and peroxidase [POD] activity and proline [PRO] and malondialdehyde [MDA] content) were measured at different stages of infection ( $12 \mathrm{hpi}$ : spore germination, $24 \mathrm{hpi}$ : infective vesicles stage, 48 hpi: intercellular infective hyphae and control). Significant differences compared with the control (the Student's $t$ test) are indicated as follows: one asterisk $(*), P<5 \times 10^{-2}$ and two $(* *), P<1 \times 10^{-2}$. Error bars represent standard error, values are presented as mean \pm standard error and $n=3$. 
process, chitin metabolic process, and cell-wall macromolecule catabolic process were enriched at 12 versus $24 \mathrm{hpi}$, and genes associated with transcription and lipid metabolic process were activated in 24 versus 48 hpi (Supplementary Data S2).

Consistent with these findings, Kyoto Encyclopedia of Genes and Genomes (KEGG) enrichment analysis revealed that the significant pathways of the upregulated DEGs in CK versus 12 hpi were mainly enriched in 'Regulation of autophagy', 'Aminobenzoate degradation', 'Glycine, serine and threonine metabolism', 'plant-pathogen interaction', 'peroxisome', and 'pentose phosphate pathway', while the downregulated DEGs were mainly enriched in 'pyruvate metabolism', 'glycolysis/ gluconeogenesis', 'carbon fixation in photosynthetic organisms', 'porphyrin and chlorophyll metabolism', 'starch and sucrose metabolism', and 'fatty acid metabolism' (Fig. 3B; Supplementary Data S3). In the 12 versus 24 hpi samples, DEGs related to 'pyruvate metabolism', 'bisphenol degradation', and 'flavonoid biosynthesis' were up-regulated, and DEGs in the pathways 'photosynthesis', 'circadian rhythmplant', and 'mineral absorption' were down-regulated (Fig. 3B; Supplementary Data S3). However, in the 24 versus 48 hpi samples, DEGs related to 'flavonoid biosynthesis', 'circadian rhythm-plant', 'antigen processing and presentation', and 'MAPK signaling pathway' were up-regulated, whereas downregulated DEGs were main enriched for the pathways 'phenylpropanoid biosynthesis', 'starch and sucrose metabolism', and 'lysosome' (Fig. 3B; Supplementary Data S3). Together, we detected dynamic transcription profiles across all three stages, suggesting that infection leads to the reprogramming of metabolism related to defense activation (Fig. 3B).

\section{Key genes associated with plant-pathogen interactions.}

To obtain a global view of the pathways involved into plantpathogen interaction, we performed MapMan analysis, which groups sets of DEGs into hierarchical functional categories based on their putative biological functions (Thimm et al. 2004) (Fig. 4A). This analysis identified 7,611 genes related to hormone signaling (auxin, salicylic acid, and jasmonic acid), cell wall, resistance $(R)$ genes, respiratory burst, TFs (MYB, WRKY, ERF, bZIP), peroxidases, and secondary metabolites that were activated in response to pathogen infection (Fig. 4A). Genes related to cell-wall synthesis (cellulose synthesis, cellwall modification) and cell-wall degradation ( $\beta$-1,4-glucanases, pectate lyases, and polygalacturonases) were activated during the first two timepoints of infection and were down-regulated at 48 hpi. Two genes involved in respiratory bursts, RBOHC (Potri.012G111600) and RBOHD (Potri.003G159800), were also activated during this process, suggesting they function in cell phagocytosis. Eighteen $R$ genes were significantly upregulated during infection, such as toll interleukin 1 receptornucleotide binding site-leucine-rich repeat (TIR-NBS-LRR) genes (Potri.004G19800, Potri.010G231200, Potri.007G053900, Potri.004G001500, Potri.005G004400, and Potri.001G345300), coiled coil (CC)-NBS-LRR genes (Potri.001G313300, Potri. 003G149800, and Potri.007G039100), and nucleotide-binding $\mathrm{N}$-acetyl-1-cysteine (NB-NAC) genes (Potri.001G083300, Potri. 001G393700, Potri.003G201800, and Potri.001G426500) (Supplementary Data S4). In total 24 WRKY and 42 MYB TF genes were significantly up-regulated during infection, including the genes encoding MYB94 (Potri.017G082500), MYB12 (Potri. 003G144300), WRKY11 (Potri.018G008500), WRKY18 (Potri. 006G263600), WRKY 33 (Potri.016G128300), and WRKY40 (Potri.018G019800). Also, 57 secondary metabolite genes were upregulated during infection. A detailed description of the results of MapMan analysis of the DEGs is provided in Supplementary Data S4. In summary, we identified many key genes involved in the plant-pathogen interaction pathway as upregulated during $M$. brunnea infection.

\section{Significant association of allelic variation in genes of the plant-pathogen interaction pathway with photosynthetic and growth traits.}

To elucidate the potential molecular mechanisms underlying plant coordination of photosynthetic and growth processes with defense responses, we explored the functions of genes and the genetic networks in the plant-pathogen interaction pathway by searching for SNPs in the DEGs, including the regions $2 \mathrm{~kb}$ upstream and $1.5 \mathrm{~kb}$ downstream of each gene. In total, we detected 29,399 common SNPs within 221 full-length genes in the plant-pathogen interaction pathway, i.e., approximately 18 SNPs per kilobase (Supplementary Data S5). We performed association analysis, using TASSEL software, based on a mixed linear model, which takes into account the kinship matrix and genetic structure $(K+Q)$. The analysis identified 2,553 significant associations $\left(P<1 \times 10^{-2}\right)$ representing 1,701 SNPs in 166 candidate genes and phenotypes, including four photosynthetic and three growth traits (Fig. 5A; Supplementary Data S6). Of these, 104 SNPs in 51 genes were associated with four photosynthetic traits, i.e., net photosynthetic rate (28 SNPs, 24 genes), stomatal conductance (Cond) (24 SNPs, 15 genes), intercellular $\mathrm{CO}_{2}$ concentration (Ci) (28 SNPs, 13 genes), transpiration rate (24 SNPs, 20 genes) (Fig. 4BC; Supplementary Fig. S2). Notably, Pt-SNH2-SNP62 was significantly associated with Cond (Fig. 4B) and explained $45.2 \%$ of the

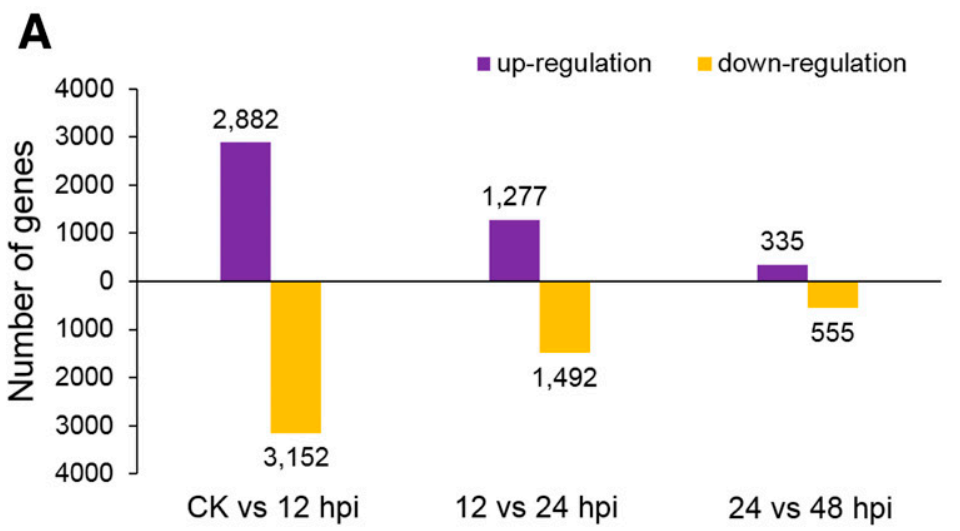

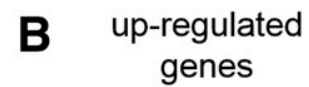

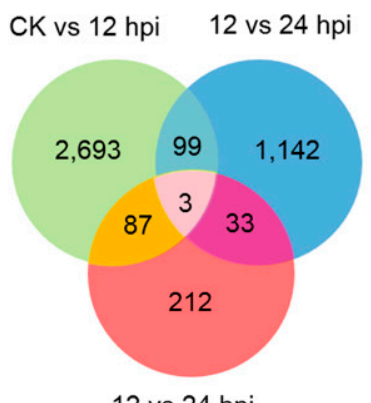

12 vs 24 hpi
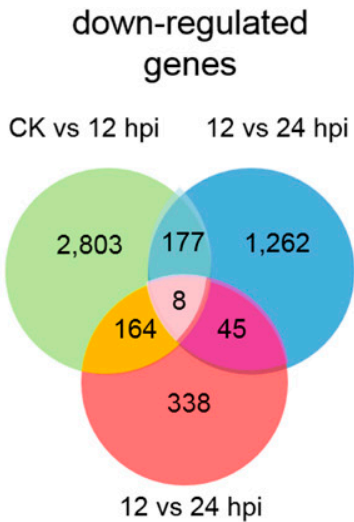

Fig. 2. Identification of genes differentially expressed during infection. A, Differentially expressed genes at different stages of infection. Up- and downregulated genes are represented by purple and orange bars, respectively. B, Comparison of differentially expressed genes at different stages of infection. Genes that were differentially expressed between CK and $12 \mathrm{~h}$ postinoculation (hpi), 12 and $24 \mathrm{hpi}$, and 24 and $48 \mathrm{hpi}$ are shown in blue, green, and red, respectively. 
phenotypic variation (the highest contribution to phenotype), suggesting it plays important roles in photosynthesis and tree growth properties. We detected 2,449 associations for 1,624 SNPs in 160 genes associated with three growth traits, including diameter at breast height $(\mathrm{DBH})(1,552$ SNPs, 155 genes), tree height $(\mathrm{H})$ (36 SNPs, 22 genes), and stem volume (V) (861 SNPs, 122 genes). A total of 1,701 SNP markers exhibited significant associations with at least one trait, and the number of significant SNPs ranged from one to three per trait. The total number of SNP-trait associations varied across trait categories, with 104 associations for photosynthetic traits and 2,449 associations for growth traits.

Marker-trait association analysis identifies genes that might be important for photosynthesis and growth processes. As expected, $R$ genes (involved in pathogen resistance) were associated with the seven traits described above (Fig. 4BC;
Supplementary Fig. S2). For instance, SNPs in two TIR-NBSLRR genes (Potri.002G056100 and Potri.017G011800) and two LRR genes (Potri.006G179400 and Potri.001G467300) were significantly associated with DBH (Supplementary Fig. S2C); 22 SNPs in an NB-ARC gene (Potri.005G062300) were significantly associated with V (Supplementary Fig. S2D); two SNPs (SNP131 and SNP139) in a protein kinase superfamily gene (Potri.001G393200) were significantly associated with $\mathrm{H}$ (Supplementary Fig. S2E). Notably, 24 SNPs in a TIR-NBSLRR gene (Potri.002G056100) were significantly associated with three growth traits $(\mathrm{H}, \mathrm{V}$, and $\mathrm{DBH})$. Moreover, SNPs in an LRR gene (Potri.006G014300) and SNH2 (Potri.018G135700) were significantly associated with Cond (Fig. 4B). Finally, SNP58 in ERF9 (Potri.017G013700) and SNP33 in ERF3 (Potri.001G356100) were significantly associated with transpiration rate (Supplementary Fig. S2B), three SNPs in

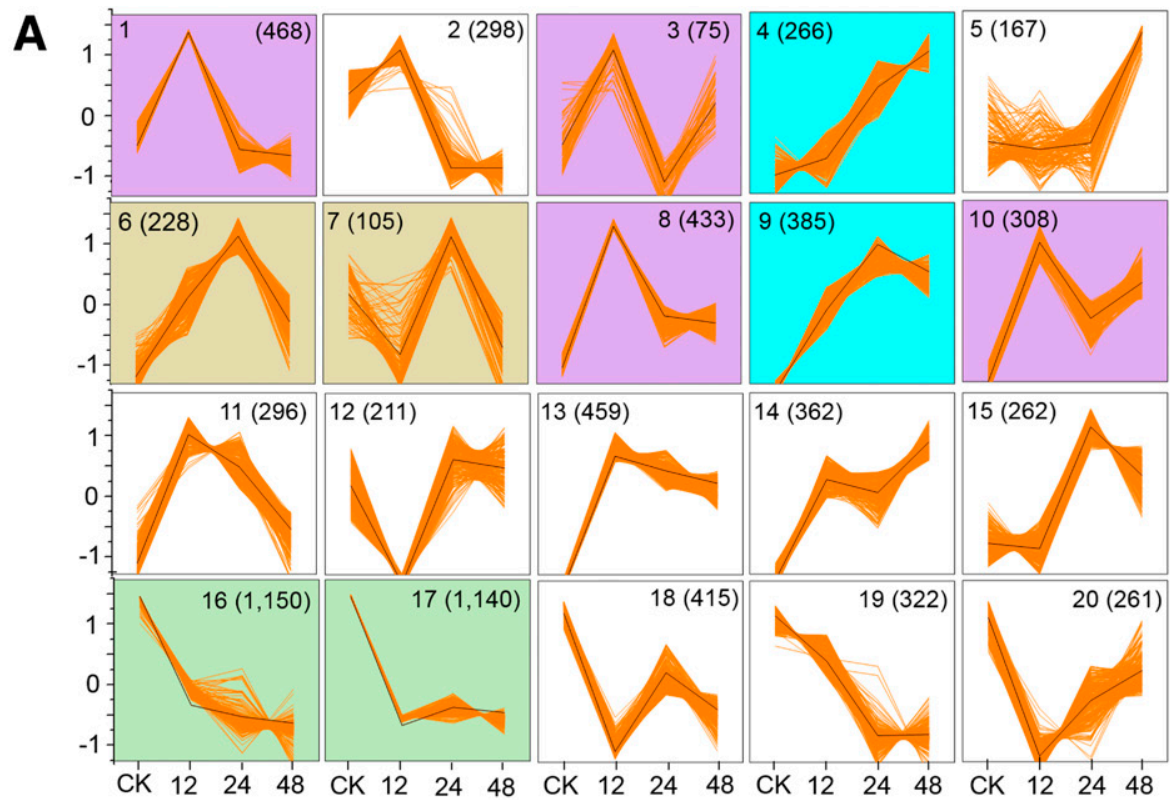

B

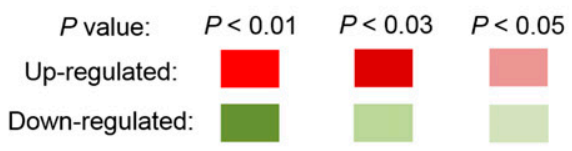

CK vs 12 hpi

Bisphenol degradation Aminobenzoate degradation

Glycine, serine and threonine metabolism

Plant-pathogen interaction

Peroxisome

Pentose phosphate pathway

Pyruvate metabolism

Glycolysis / Gluconeogenesis

Carbon fixation in photosynthetic organisms

Porphyrin and chlorophyll metabolism

Ascorbate and aldarate metabolism

Starch and sucrose metabolism

Fatty acid metabolism

Biosynthesis of unsaturated fatty acids
12 hpi vs 24 hpi

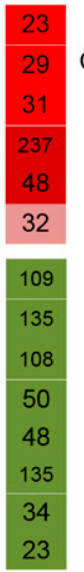

$\begin{array}{rcc}\text { Pyruvate metabolism } & 53 \\ \text { Carbon fixation in photosynthetic organisms } & 57 \\ \text { Glycolysis / Gluconeogenesis } & 59 \\ \text { Bisphenol degradation } & 12 \\ \text { Oxidative phosphorylation } & 30 \\ \text { Flavonoid biosynthesis } & 9 \\ \text { Photosynthesis - antenna proteins } & 27 \\ \text { Photosynthesis } & 34 \\ \text { Circadian rhythm - plant } & 18 \\ \text { Cell cycle } & 29 \\ \text { Biosynthesis of unsaturated fatty acids } & 17 \\ \text { Mineral absorption } & 5 \\ \text { Terpenoid backbone biosynthesis } & 18 \\ \text { Porphyrin and chlorophyll metabolism } & 21\end{array}$

24 hpi vs 48 hpi

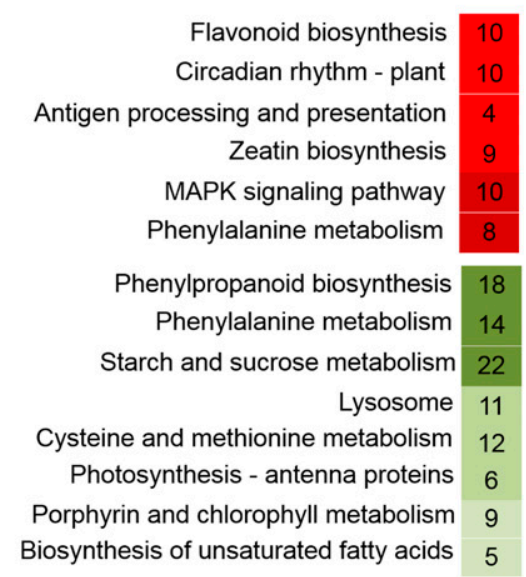

Fig. 3. Transcriptome dynamics at different stages of infection. A, Twenty clusters obtained by $K$-means clustering with Euclidean distance as the distance metric. B, Significant pathways of the up- and downregulated differentially expressed genes at different stages of infection. The intensity of the color represents the significance of the $P$ value (red for upregulated, blue for downregulated). The values shown represent the number of genes enriched in each pathway. 
CC-NBS-LRR (Potri.005G061800 and Potri.007G123200) and bHLH genes (Potri.012G106000) were significantly associated with photosynthetic rate (Supplementary Fig. S2A), and Pt-LRR-SNP98 (Potri.008G034000), and seven SNPs in CRINKLY4 (Potri.001G252400) were significantly associated with $\mathrm{Ci}$ (Fig. 4C). These significant marker-trait associations indicate that these genes in the plant-pathogen interaction pathway play important roles in photosynthesis and growth.

\section{Genes in functional modules}

in the plant-pathogen interaction pathway show significant epistatic interactions.

We examined potential epistatic interactions between SNPs in genes in the plant-pathogen interaction pathway associated with photosynthetic and growth traits using the epiSNP package, which can identify SNP-SNP interactions in a population. Three traits $(\mathrm{H}, \mathrm{DBH}$, and Cond traits) had significant epistatic interactions $\left(P<1 \times 10^{-3}\right)$. In total, we detected 4,460 significant associations representing 805 unique SNPs within 115 genes associated with these three traits $\left(P<1 \times 10^{-3}\right.$; Supplementary Data S7). These 4,460 pairwise epistatic interactions were further categorized into 921 additive $\times$ additive, 2,311 additive $\times$ dominance or dominance $\times$ additive, and 1,228 dominance $\times$ dominance effects for the traits (Supplementary Data S7). Interestingly, epistatic interactions were most common for Cond, reaching 4,390 SNP pairs, whereas only 47 and 23 pairs of epistatic interactions were identified for $\mathrm{H}$ and $\mathrm{DBH}$, respectively.

To obtain a global view of the roles of these epistatically interacting genes in plant defense responses, photosynthesis, and growth, we grouped the genes into eight functional modules (groups A to $\mathrm{H}$ : $\mathrm{A}=$ hormone signaling, $\mathrm{B}=$ cell wall, $\mathrm{C}=$ $R$ gene, $\mathrm{D}=$ signaling, $\mathrm{E}=\mathrm{MAPK}, \mathrm{F}=\mathrm{TFs}, \mathrm{G}=$ secondary metabolites, $\mathrm{H}=$ respiratory burst) (Fig. 5B; Supplementary Data S8) based on MapMan analysis. Statistical analysis revealed eight functional modules that genetically interacted with each other as well as 2,561 associations between functional modules (Fig. 5B). Of the SNP-SNP pairs associated with the Cond trait, 377 SNP-SNP pairs were detected in genes from
A
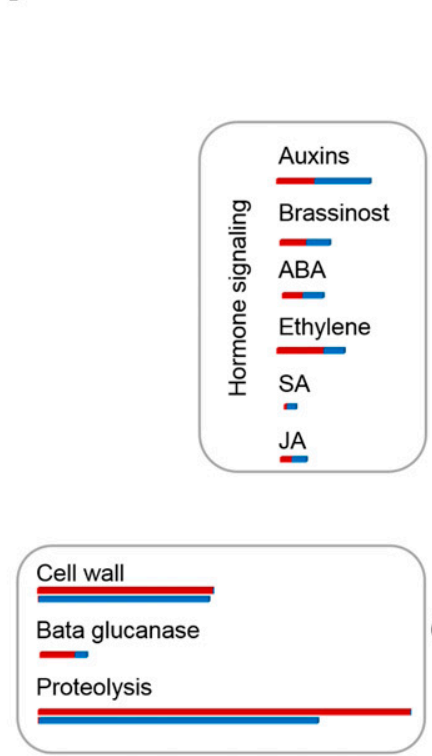

B

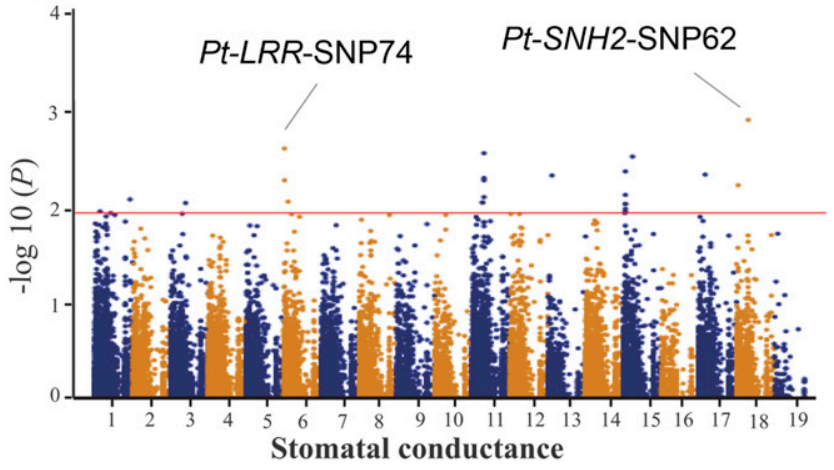

Pathogen attack

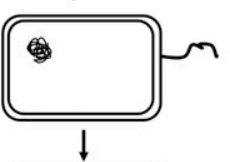

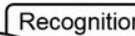

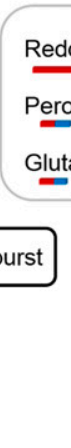

Redox stat

Peroxidses

Glutathione-S-transferase

MAPK
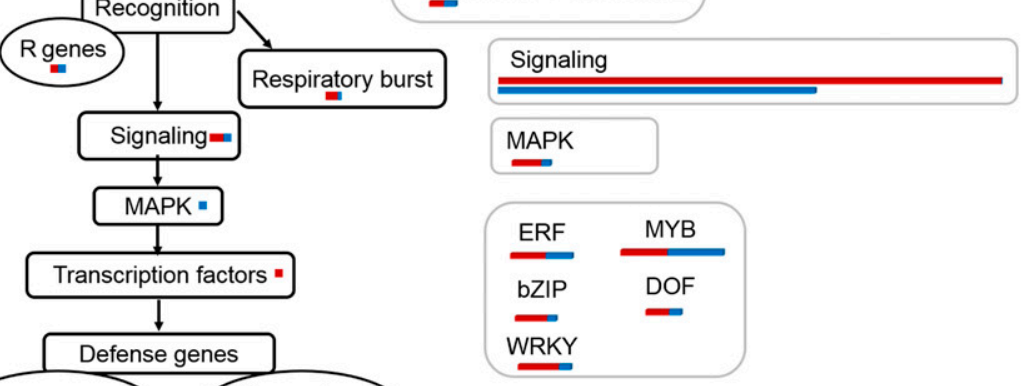

- Red: up-regulated gene

- Green: down-regulated gene

Heat shock proteins

Secondary metabolites

C

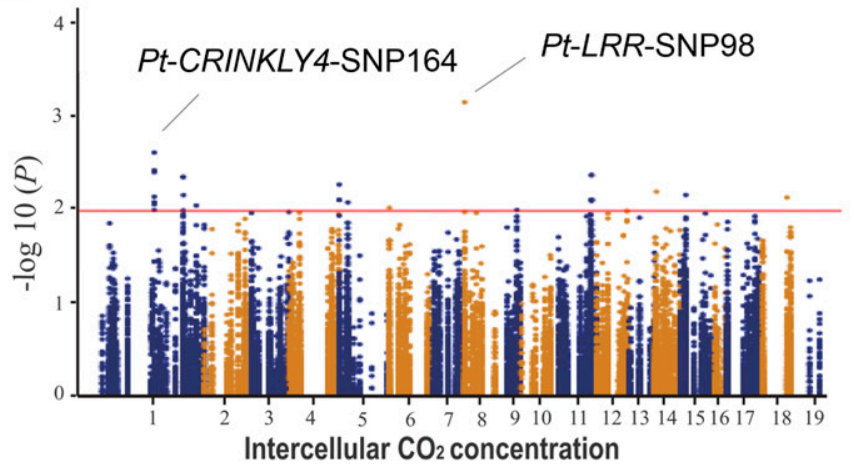

Fig. 4. Differentially expressed genes (DEGs) involved in plant-pathogen interactions. A, MapMan analysis illustrating the DEGs involved in plant-pathogen interactions. The 'plant-pathogen interaction pathway' was used to visualize transcriptional changes in genes with putative functions. Genes that were upregulated by the treatments are shown in red and those that were down-regulated are shown in blue. The length of the bars indicates the number of genes. $\mathbf{B}$ and C, Genome distribution of significant associations for the stomatal conductance and intercellular $\mathrm{CO}_{2}$ concentration traits in the association population of Populus tomentosa. The red line in each Manhattan plot depicts the Bonferroni-adjusted significance threshold $\left(1 \times 10^{-2}\right)$. The $x$ and $y$ axes show the genomic position and the significance denoted as $\log _{10}(P)$, respectively. 
groups $\mathrm{C}$ and $\mathrm{D}\left(P<1 \times 10^{-3}\right)$ and 88 SNP-SNP pairs $(P<1 \times$ $10^{-3}$ ) were detected in genes from groups $\mathrm{C}$ and $\mathrm{F}$. Multiway SNP-SNP interaction analysis showed that SNPs had numerous epistatic interactions between the genes in group D and other groups. (Fig. 5B). Of these genes, 30.4\% had epistatic interactions with genes in group A, $76.8 \%$ with genes in group $\mathrm{C}$, $60.7 \%$ with genes in group $\mathrm{F}$, and $10.7 \%$ with genes in group $\mathrm{H}$. Based on these findings, we constructed a proposed epistatic network among genetic factors in the biotic stress pathway, revealing the synergistic interactions between the eight functional modules.

\section{DISCUSSION}

Plant photosynthesis and growth are often inhibited by defense responses (Erickson et al. 2004). The expression of induced plant defenses is mediated by complex defense networks and requires a vast interaction of genes and the cooperation of processes. Confirming the phases of different processes has an important significance in investigating plant defense and guiding the operating of breeding systems of natural populations in response to biotic stress, for such variation will provide powerful markers for upstream factors that act in plant defense networks. We constructed a time series transcriptome atlas covering the major transitions of $P$. tomentosa leaves infected by M. brunnea. Global comparisons of gene expression highlighted the fundamental transcriptomic reprogramming and well-demonstrated plant defense pathways present. Our results show that at least 7,611 genes, including $628 \mathrm{TFs}$, are required to program the defense process, which involves about $18.41 \%$ of the poplar genes. By using multigene association analysis, we identified 29,399 common SNPs within 221 full-length genes in plant-pathogen interaction pathways that were significantly associated with photosynthetic and growth traits. Epistasis analysis uncovered significant interactions between 2,561 SNP-SNP pairs from different functional modules in the plant-pathogen interaction pathway, revealing possible genetic interactions. Management and modification of the key genes and these genetic markers will affect plant fitness, which is influenced by genetic variation in trees (Whitham et al. 2008).

\section{Transcriptome reprogramming at different stages of the infection process.}

Plants have adapted to tolerate and survive under constantly changing environmental conditions, including a variety of abiotic and biotic stresses, by reprogramming the expression of genes involved in photosynthetic and growth processes and crucial metabolic processes (Tufan et al. 2009). Plants and pathogens constantly confront each other in a battle for growth and survival, and the outcome of such encounters in agriculturally important species can directly interfere with crop production (Karasov et al. 2017). To cope with pathogen stress, plants exhibit dynamic, tightly regulated transcriptomic changes (Kistner et al. 2005; Liu et al. 2015; Mine et al. 2018; Teixeira et al. 2014; Tufan et al. 2009). Immune signaling in plants involves both positive and negative regulators. Maintaining a balance between growth and defense responses is crucial to appropriate environmental responses (Sreekanta 2014). In the current study, significant dynamic transcriptome changes were detected during the infection of poplar with M. brunnea. Genes involved in photosynthesis were repressed at the infective vesicles stage (24 hpi) and in intercellular infective hyphae (48 hpi), indicating that photosynthetic capacity decreases upon fungal infection. The reduction in photosynthesis upon fungal infection is an adaptive strategy used by plants to switch off assimilation processes in favor of respiration processes so as to generate products required for defense (Luo et al. 2019; Major et al. 2010; Scholes and Rolfe 1996).

Conversely, genes involved in plant-pathogen interaction, the MAPK signaling pathway, and apoptosis were activated during M. brunnea infection. These pathways represent important molecular mechanisms involved in plant disease resistance. Meanwhile, genes related to starch and sucrose metabolism and biosynthesis of unsaturated fatty acids were significantly repressed during infection. Reduced photosynthetic capacity and the biosynthesis of organic compounds eventually lead to growth restrictions, which may help plants improve their defense abilities upon pathogen attack. The extensive functional changes across different timepoints demonstrate a much higher degree of complexity in the regulation of plant responses to infection than had been previously estimated by simply performing mRNA analysis of DEGs (Chang et al. 2018).
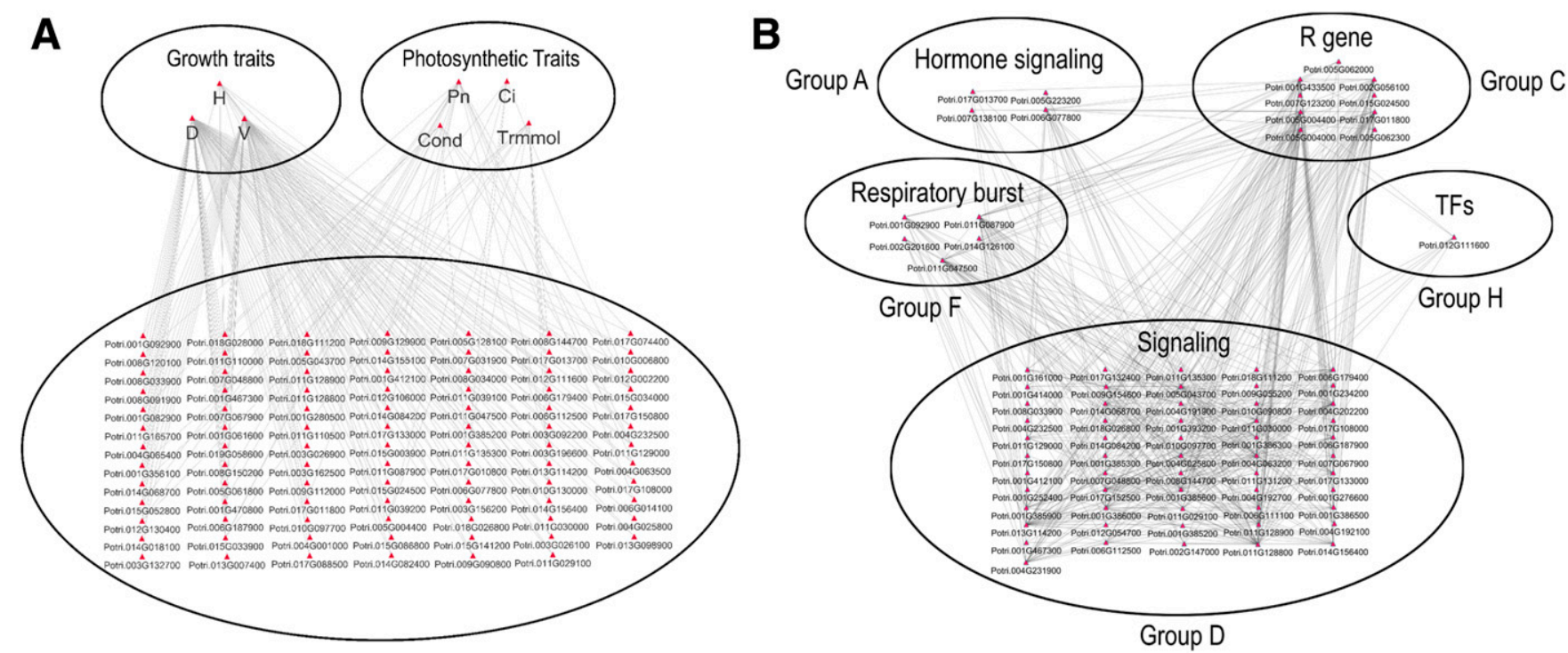

Fig. 5. Summary of genetic interactions among genes in the plant-pathogen pathway. A, Association between single-nucleotide polymorphisms in genes of the plant-pathogen pathway with tree growth and photosynthetic traits. Black triangles represent traits and red triangles represent genes. B, Epistatic interactions between functional modules in the plant-pathogen interaction pathway. 


\section{Key genes involved in the defense response.}

Plants have evolved sophisticated mechanisms for disease resistance involving the recognition and rapid response to pathogen molecules. We identified genes with transient, adaptive, and dynamic expression profiles in response to infection, indicating key roles in defense responses. The massive changes in expression of thousands of defense-responsive genes, including disease- $R$ genes and TF genes, reflects the activation of transcriptional responses to pathogen infection. $R$ genes protect plants from pathogen invasion. Some $R$ genes recognize microorganisms at the cell surface, to initiate pathogen-associated molecular pattern-triggered immunity by plant pattern recognition receptors, which usually prevents the plant from becoming infected before the microbe gains hold in the plant. Some $R$ genes recognize effector proteins secreted by successful pathogens through plant $\mathrm{R}$ proteins in a highly specific manner (Dodds et al. 2006). This type of specific recognition induces effector-triggered immunity that is crucial for pathogens expressing cognate avirulence genes and initiating defense responses leading to resistance (Moffett 2009; Rajamuthiah and Mylonakis 2014). Most $R$ genes play indirect roles in proteinprotein interactions during inducible resistance responses (van Loon 1985) and mainly function in the cytoplasm (Dodds and Rathjen 2010; Tameling and Baulcombe 2007; Weaver et al. 2006). The NB-LRR proteins are the major proteins encoded by $R$ genes (Weaver et al. 2006). Indeed, we detected numerous $R$ genes belonging to the CC-NBS-LRR, TIR-NBS-LRR, and NB-NAC classes that were highly expressed during $M$. brunnea infection. This finding suggests that these $R$ genes, whose products function as cytosolic resistance proteins, delay or halt pathogen progress.

Genes encoding several TFs were activated during $M$. brunnea infection, implying that they function in defense responses in poplar. $P t-W R K Y 18$ and $P t$-WRKY40 were upregulated after infection (12 to $48 \mathrm{hpi}$ ), suggesting they have important roles in the basal defense response in Populus spp. Indeed, WRKY18/40 negatively affects pre-invasion host defense responses and regulates a subset of defense genes (Pandey et al. 2010). The R2R3-type MYB94, an activator of cuticular wax biosynthesis, is essential in plant defense (Lee and Suh 2015). Consistent with this finding, $P t-M Y B 94$ expression was high at 12 to $24 \mathrm{hpi}$ and decreased to a low level thereafter. PtMYB12 was also activated in our dataset; MYB12 regulates phenylalanine biosynthesis, which might play a key role in disease resistance (Luo et al. 2008; Mehrtens et al. 2005). The speed of induction of infection-induced TFs suggests that transcriptional responses are a major driver of transcriptome reprogramming. Future work should address the functions of these putative regulators of plant defense responses.

\section{Functional interpretation}

\section{of genetic associations and epistasis.}

Plants are constantly exposed to threats from pathogens and have evolved complex mechanisms to recognize and defend themselves against attack (Rojas et al. 2014). Defense responses include changes in primary metabolic pathways, hormone signaling pathways, cell-wall fortification, cytoskeletal reorganization (Hardham et al. 2007; Higaki et al. 2011), transcription of pathogenesis-related proteins (van Loon et al. 2006), and the hypersensitive response (Coll et al. 2011). However, assessing the effects of functional modules in the plant-pathogen interaction pathway on plant defense and their potential effects on growth using traditional molecular genetic methods has always been challenging. In the current study, we used multigene association approaches combining the analysis of 221 full-length candidate genes in functional modules in the plant-pathogen interaction pathway to identify 2,553 loci potentially associated with photosynthesis and growth traits. This SNP-based association mapping method is suitable for annotating gene functions and identifying genetic regulatory networks and is useful for exploring the functions and interactions of genes in the plant-pathogen interaction pathway. One finding of interest was that, although the number of candidate genes associated with the Cond trait was not the highest among the categories, these genes had the most significant effects on phenotype. For example, SNH2 (Pt-SNH2-SNP62) made the greatest contribution to phenotypic variation $(45.2 \%)$. The significant association between these genes and the Cond trait suggests that they might function in coordinating stomatal movement with photosynthesis and plant growth (Gortari et al. 2018).

Epistasis analysis is extensively used to decipher the functional relationships and genetic interactions between genes or regulatory modules in the same pathway, particularly in organisms with natural populations in which large numbers of mutants can be generated (Phillips 2008), such as poplar. In the current study, we identified 4,460 significant SNP-SNP pairs $\left(P<1 \times 10^{-3}\right)$ in 94 genes related to different processes associated with seven traits, reflecting possible genetic interactions and potential target candidate genes. Moreover, we grouped epistatic interactions into eight hierarchical functional categories, with MapMan, to elucidate the potential biological functions of the epistatically interacting genes. Significant SNP-SNP interactions $\left(P<1 \times 10^{-3}\right)$ between genes in groups $\mathrm{A}, \mathrm{C}, \mathrm{D}$, and $\mathrm{F}$ were detected, suggesting that these may have roles in the same pathways. At least $22.2 \%$ of the 18 genes in group C ( $R$ gene) had epistatic interactions with genes in group $\mathrm{A}, \mathrm{D}$, and $\mathrm{F}$, suggesting that these genes function in plant growth and survival. SNP-SNP pairs $\left(P<1 \times 10^{-3}\right)$ in genes from groups $\mathrm{C}$ and $\mathrm{D}$ and groups $\mathrm{C}$ and $\mathrm{F}$ were only detected for the Cond trait, highlighting the role of stomata in regulating plant disease resistance, photosynthesis, and growth.

In total, 98.43\% SNP-SNP pairs, representing 4,390 gene pairs, were associated with Cond. Stomata, the sites of gas exchange and water transpiration, function in innate immunity by limiting pathogen entry by actively closing, the so-called stomatal defense response (Melotto et al. 2017). These findings suggest that the coselection of genes in functional modules, genetic interactions, or protein-protein interactions might represent an essential mechanism that protects plants from pathogens. The incorporation of more key genes (encoding transporters or receptors) in shared regulatory pathways would provide a more complete picture of the effects of genetic interactions on plant defense pathways.

\section{MATERIALS AND METHODS}

Plant materials and strains.

Populus tomentosa cv. LM50 clones from the Poplar Seedling Station of the Poplar Experimental Bureau (Guanxian County, Liaocheng City, Shandong Province, China) were used in this study. One-year-old $P$. tomentosa LM50 clones were cultured in a greenhouse maintained at $25 \pm 2{ }^{\circ} \mathrm{C}$ under $12-\mathrm{h}$ light conditions. The fungal strain used in this study was Marssonina brunnea f. sp. monogermtubi bj01 isolated from P. tomentosa in Beijing Forestry University. For isolation, leaf pieces infected by $M$. brunnea were washed with sterile water, were sterilized with $75 \%$ alcohol for $30 \mathrm{~s}$, and were incubated in sterile $2 \%$ agar culture plates at $25^{\circ} \mathrm{C}$ for 2 days to produce white conidia. The conidia were transferred to potato dextrose agar medium containing $50 \mu \mathrm{g}$ of streptomycin sulfate per milliliter, using a sterile inoculating needle.

\section{Inoculation and sample collection.}

Healthy, fully expanded poplar leaves were collected, were sterilized in $75 \%$ ethanol for $20 \mathrm{~s}$, were washed three times with 
sterile water, and were plated onto sterile $2 \%$ agar culture plates with the abaxial surface of the leaf facing upward. $M$. brunnea conidia were suspended in sterile water and the concentration of the suspension was adjusted to approximately $5 \times 10^{5}$ spores per milliliter. The conidia were sprayed onto the abaxial surfaces of the leaves. The inoculated leaves were incubated in an artificial climate incubator (LT36VL; Percival Scientific, Inc.) at $95 \%$ relative humidity, $25^{\circ} \mathrm{C}$ with a 12 -h photoperiod and were harvested at 12, 24, and $48 \mathrm{hpi}$. In the CK, the leaves were sprayed with sterile tap water and were harvested at 12 hpi. Samples of infected leaves subjected to the same conditions (treatment and timepoint) were pooled and were treated as one biological repeat, and three repeats were performed per treatment (CK and 12, 24, and $48 \mathrm{hpi}$ ). All leaf samples were immediately frozen in liquid nitrogen and stored at $-80^{\circ} \mathrm{C}$ for RNA extraction.

\section{RNA extraction and transcriptome sequencing.}

Total RNA was isolated from the samples using TRIzol reagent (Invitrogen) according to manufacturer instructions. The quality of RNA was checked by agarose gel electrophoresis to ensure that clear bands were visible. The RNA integrity number of each sample was determined using a NanoDrop 2000 spectrophotometer (Thermo Scientific). Samples of sufficient quality were sent for RNA-seq cDNA library construction and were sequenced on the Illumina HiSeq 4000 (Illumina, Inc.) sequencing platform on a flow cell. The raw sequence data reported in this paper have been deposited and are publicly accessible in the Genome Sequence Archive in the Beijing Institute of Genomics BIG Data Center, Chinese Academy of Sciences, under accession number CRA001647. The RNA samples were stored at $-80^{\circ} \mathrm{C}$ for further gene expression analysis.

\section{qPCR validation of expression profiles.}

To verify the RNA-seq results, nine genes were selected to examine the consistency of gene expression profiles between the RNA-Seq and qPCR assays of M. brunnea-infected leaves (Supplementary Fig. S1). The RNA samples were treated with DNase I (TaKaRa), and a PrimeScript RT reagent kit (Perfect Real Time) with gDNA Eraser (Takara) was used for cDNA synthesis with oligo (dT) as the primer. The 7500 Fast RealTime PCR System (Applied Biosystems) and SYBR Green Premix Ex Taq (TaKaRa) were used for all qPCR assays. All PCRs were performed using the two-step qPCR procedure, and three replications per sample were performed to reduce the experimental error. The relative expression levels of the genes based on qPCR were calculated using the ${ }^{\Delta \Delta} \mathrm{CT}$ method (Livak and Schmittgen 2001). The genes and corresponding primers are listed in Supplementary Table S2.

\section{Quality control and identification \\ of differentially expressed transcripts.}

The raw RNA-seq data were quality-filtered using fastp software with default parameters (Chen et al. 2018b). The quality control of raw reads process included: i) removing paired reads with adapters; ii) removing paired reads when the $\mathrm{N}$ content in any sequencing read $>10 \%$ of the read bases; iii) removing paired reads containing $>50 \%$ low-quality nucleotides ( $\mathrm{sQ} \leq 5)$. Clean reads were mapped to the v3.0 genome of Populus trichocarpa using Hisat2 (v2.0.9) with the parameter $-q-x-S-p$. to generate read alignments for each sample (Kim et al. 2013). To identify DEGs in poplar in response to $M$. brunnea infection at different timepoints after inoculation, a two-step analysis was carried out. First, fragments per kilobase per million mapped reads (FPKM) values were calculated, to measure the abundance and expression of each transcript using
Cufflinks software (Ghosh and Chan 2016; Trapnell et al. 2010). Second, differences in gene expression among samples (12, 24, and 48 hpi and CK) were detected using Cuffdiff. Cuffdiff was used to identify up- and downregulated genes by comparing the expression levels of transcripts under two conditions (CK versus $12 \mathrm{hpi}, 12$ versus $24 \mathrm{hpi}$, and 24 versus $48 \mathrm{hpi}$ ). The DEGs identified from the three infected timecourses were filtered using the parameters $P$ value $\leq 5 \times 10^{-2}$ and $\log _{2} \mathrm{FC} \geq 1$ (upregulated genes) or $\leq-1$ (downregulated genes) to judge significant differences in gene expression.

\section{DEG analysis and functional annotation.}

To identify overlapping up- and downregulated DEGs, Venn diagrams were constructed using the Draw Venn Diagram tool. Gene clustering analysis was performed using the $K$-means partitioning method in the MultiExperiment Viewer package version 4.9.0 (Howe et al. 2010).

GO functional enrichment analysis of the DEGs was performed using AgriGO version 2.0. For pathway analysis, all DEGs were mapped to KEGG pathways, and significantly enriched pathways with $P$ value $\leq 5 \times 10^{-2}$ were identified. The results of gene enrichment analysis of DEGs in the biotic stress pathway were visualized using MapMan version 3.5.1 (Thimm et al. 2004).

\section{Association analysis of DEGs}

\section{in the plant-pathogen interaction pathway.}

Association population and phenotypic data. An association population of 435 unrelated $P$. tomentosa individuals (randomly selected from a clonal arboretum) was used for SNP-based association studies. Four photosynthetic traits and three growth traits were measured from all individuals in the association population, with at least three replications per genotype. The data for photosynthetic and growth traits were collected as described by Wang et al. (2017) and Du et al. (2015) (Supplementary Data S9). Photosynthetic traits, including net photosynthetic rate, Cond, $\mathrm{Ci}$, and transpiration rate, were measured in fully expanded leaves using the LI-6400 portable photosynthesis system (LI-COR Inc.). Growth traits, including DBH, H, and $\mathrm{V}$, were measured from the apex to the base of the tree, and DBH was measured using Vernier calipers. In this study, four photosynthetic traits and three growth traits of the above population were used to associate with the genes in the plantpathogen interaction pathway.

SNP discovery and genotyping. The 435 unrelated $P$. tomentosa individuals from the natural population were resequenced at a depth $>15 \times$ (raw data), using the Illumina GA II platform. Filtered reads were mapped to Populus reference genome v3.0 (Tuskan et al. 2006), using SOAPaligner (SOAP2, version 2.20) with default options ( $\mathrm{Li}$ et al. 2009), which were used for SNP calling. To obtain high-quality SNPs, SNPs were called using selected uniquely mapped paired end reads. The genotype data based on gene-derived biallelic SNPs within the full-length sequences, including $2 \mathrm{~kb}$ of upstream and $1.5 \mathrm{~kb}$ of downstream sequence, were extracted using VCFtools (Danecek et al. 2011) according to the annotation of the Populus genome (version 3.0) download from Phytozome. The genotypic data of the 435 individuals are shown in Supplementary Data S10.

Epistatic interaction analysis. All trait-SNP association analyses between SNPs and the four photosynthetic traits and three growth traits were performed using the software package TASSEL v5.0 (Bradbury et al. 2007) based on the mixed linear model, accounting for the population structure $(Q)$ and pairwise kinship coefficients $(K)$. The $K$ matrix was obtained using TASSEL, and the $Q$ matrix of the covariates was identified as described by Du et al. (2012). EpiSNP software (Murase et al. 
2008) was used to detect two-locus epistatic interactions based on the extended Kempthorne model. All $P$ values were corrected using Benjamini-Hochberg methods in $\mathrm{R}$, and the significance levels of the $P$ value for the association analysis results were controlled at $1 \times 10^{-2}$.

\section{AUTHOR-RECOMMENDED INTERNET RESOURCES}

AgriGO tool and database: http://systemsbiology.cau.edu.cn/agriGOv2 Draw Venn Diagram tool:

http://bioinformatics.psb.ugent.be/webtools/Venn

Genome Sequence Archive: http://bigd.big.ac.cn/gsa

Phytozome: http://www.phytozome.net

\section{LITERATURE CITED}

Afzal, F., Khurshid, R., Ashraf, M., and Kazi, A. G. 2014. Reactive oxygen species and antioxidants in response to pathogens and wounding. Pages 397-424 in: Oxidative Damage to Plants. Elsevier, New York.

Azevedo, R. F., Gonçalves-Vidigal, M. C., Oblessuc, P. R., and Melotto, M. 2018. The common bean COK-4 and the Arabidopsis FER kinase domain share similar functions in plant growth and defence. Mol. Plant Pathol. 19:1765-1778.

Balasubramanian, S., Zheng, D., Liu, Y.-J., Fang, G., Frankish, A., Carriero, N., Robilotto, R., Cayting, P., and Gerstein, M. 2009. Comparative analysis of processed ribosomal protein pseudogenes in four mammalian genomes. Genome Biol. 10:R2.

Bradbury, P. J., Zhang, Z., Kroon, D. E., Casstevens, T. M., Ramdoss, Y., and Buckler, E. S. 2007. TASSEL: Software for association mapping of complex traits in diverse samples. Bioinformatics 23 : 2633-2635.

Chang, H. X., Sang, H., Wang, J., McPhee, K. E., Zhuang, X., Porter, L. D. and Chilvers, M. I. 2018. Exploring the genetics of lesion and nodal resistance in pea (Pisum sativum L.) to Sclerotinia sclerotiorum using genome-wide association studies and RNA-Seq. Plant Direct 2:e00064.

Chen, C., Yao, Y., Zhang, L., Xu, M., Jiang, J., Dou, T., Lin, W., Zhao, G., Huang, M., and Zhou, Y. 2015. A comprehensive analysis of the transcriptomes of Marssonina brunnea and infected poplar leaves to capture vital events in host-pathogen interactions. PLoS One 10: e0134246.

Chen, F., Hu, Y., Vannozzi, A., Wu, K., Cai, H., Qin, Y., Mullis, A., Lin, Z., and Zhang, L. 2018a. The WRKY transcription factor family in model plants and crops. Crit. Rev. Plant Sci. 36:311-335.

Chen, S., Zhou, Y., Chen, Y., and Gu, J. 2018b. fastp: An ultra-fast all-inone FASTQ preprocessor. Bioinformatics 34:i884-i890.

Cheng, Q., Cao, Y., Jiang, C., Xu, L., Wang, M., Zhang, S., and Huang, M. 2010. Identifying secreted proteins of Marssonina brunnea by degenerate PCR. Proteomics 10:2406-2417.

Chisholm, S. T., Coaker, G., Day, B., and Staskawicz, B. J. 2006. Hostmicrobe interactions: Shaping the evolution of the plant immune response. Cell 124:803-814.

Coll, N. S., Epple, P., and Dangl, J. L. 2011. Programmed cell death in the plant immune system. Cell Death Differ. 18:1247-1256.

Danecek, P., Auton, A., Abecasis, G., Albers, C. A., Banks, E., DePristo, M. A., Handsaker, R. E., Lunter, G., Marth, G. T., Sherry, S. T., McVean, G., Durbin, R., and 1000 Genomes Project Analysis Group. 2011. The variant call format and VCFtools. Bioinformatics 27: 2156-2158

Dodds, P. N., Lawrence, G. J., Catanzariti, A. M., Teh, T., Wang, C. I. A., Ayliffe, M. A., Kobe, B., and Ellis, J. G. 2006. Direct protein interaction underlies gene-for-gene specificity and coevolution of the flax resistance genes and flax rust avirulence genes. Proc. Natl. Acad. Sci. U.S.A. 103: $8888-8893$

Dodds, P. N., and Rathjen, J. P. 2010. Plant immunity: Towards an integrated view of plant-pathogen interactions. Nat. Rev. Genet. 11: 539-548.

Du, Q., Lu, W., Quan, M., Xiao, L., Song, F., Li, P., Zhou, D., Xie, J., Wang, L., and Zhang, D. 2018. Genome-wide association studies to improve wood properties: Challenges and prospects. Front. Plant Sci. 9:1912.

Du, Q., Tian, J., Yang, X., Pan, W., Xu, B., Li, B., Ingvarsson, P. K., and Zhang, D. 2015. Identification of additive, dominant, and epistatic variation conferred by key genes in cellulose biosynthesis pathway in Populus tomentosa. DNA Res. 22:53-67.

Du, Q., Wang, B., Wei, Z., Zhang, D., and Li, B. 2012. Genetic diversity and population structure of Chinese white poplar (Populus tomentosa) revealed by SSR markers. J. Hered. 103:853-862.
Duplessis, S., Major, I., Martin, F., and Séguin, A. 2009. Poplar and pathogen interactions: Insights from Populus genome-wide analyses of resistance and defense gene families and gene expression profiling. Crit Rev. Plant Sci. 28:309-334.

Erickson, J. E., Stanosz, G. R., and Kruger, E. L. 2004. Photosynthetic consequences of Marssonina leaf spot differ between two poplar hybrids. New Phytol. 161:577-583.

Ghosh, S., and Chan, C.-K. K. 2016. Analysis of RNA-Seq data using TopHat and Cufflinks. Pages 339-361 in: Plant Bioinformatics, Springer, Dordrecht, The Netherlands.

Gortari, F., Guiamet, J. J., and Graciano, C. 2018. Plant-pathogen interactions: Leaf physiology alterations in poplars infected with rust (Melampsora medusae). Tree Physiol. 38:925-935.

Hamel, L., Benchabane, M., Major, I. T., Nicole, M., Sheen, J., and Séguin, A. 2011. The genomics of poplar-rust interactions to improve tree resistance against fungal disease. BMC proceedings 5:I12.

Han, Z., Yin, T., Li, C., Huang, M., and Wu, R. 2000. Host effect on genetic variation of Marssonina brunnea pathogenic to poplars. Theor. Appl. Genet. 100:614-620.

Hardham, A. R., Jones, D. A., and Takemoto, D. 2007. Cytoskeleton and cell wall function in penetration resistance. Curr. Opin. Plant Biol. 10: 342-348.

Heather, W. A., Anselmi, N., and Cellerino, C. P. 1975. In vitro and in vivo germination of spores of Marssonina brunnea (Ell. et Ev.) P. Magn. Eur. J. Forest. Pathol. 5:321-328

Higaki, T., Kurusu, T., Hasezawa, S., and Kuchitsu, K. 2011. Dynamic intracellular reorganization of cytoskeletons and the vacuole in defense responses and hypersensitive cell death in plants. J. Plant Res. 124 315-324.

Howe, E., Holton, K., Nair, S., Schlauch, D., Sinha, R., and Quackenbush, J. 2010. MeV: Multiexperiment viewer. Pages 267-277 in: Biomedical Informatics for Cancer Research. Springer, Dordrecht, The Netherlands.

Jin, H. 2016. Plant biotic interactions. J. Integr. Plant Biol. 58:282-283.

Jones, J. D., and Dangl, J. L. 2006. The plant immune system. Nature 444 323-329.

Karasov, T. L., Chae, E., Herman, J. J., and Bergelson, J. 2017. Mechanisms to mitigate the trade-off between growth and defense. Plant Cell 29: 666-680.

Kim, D., Pertea, G., Trapnell, C., Pimentel, H., Kelley, R., and Salzberg, S. L. 2013. TopHat2: Accurate alignment of transcriptomes in the presence of insertions, deletions and gene fusions. Genome Biol. 14: R36.

Kistner, C., Winzer, T., Pitzschke, A., Mulder, L., Sato, S., Kaneko, T., Tabata, S., Sandal, N., Stougaard, J., Webb, K. J., Szczyglowski, K., and Parniske, M. 2005. Seven Lotus japonicus genes required for transcriptional reprogramming of the root during fungal and bacterial symbiosis. Plant Cell 17:2217-2229.

Kutter, C., Watt, S., Stefflova, K., Wilson, M. D., Goncalves, A., Ponting, C. P., Odom, D. T., and Marques, A. C. 2012. Rapid turnover of long noncoding RNAs and the evolution of gene expression. PLoS Genet. 8 : e1002841.

Lee, S. B., and Suh, M. C. 2015. Cuticular wax biosynthesis is up-regulated by the MYB94 transcription factor in Arabidopsis. Plant Cell Physiol. 56:48-60

Li, C. 1984. Two specialized forms of Marssonina populi (Lib.) Magn. Abstract. J. Nanjing For. Univ., Nat. Sci. Ed. 4:10-17. (In Chinese).

Li, R., Yu, C., Li, Y., Lam, T. W., Yiu, S. M., Kristiansen, K., and Wang, J. 2009. SOAP2: An improved ultrafast tool for short read alignment Bioinformatics 25:1966-1967.

Liu, X., Zhou, S., Wang, W., Ye, Y., Zhao, Y., Xu, Q., Zhou, C., Tan, F., Cheng, S., and Zhou, D. X. 2015. Regulation of histone methylation and reprogramming of gene expression in the rice inflorescence meristem. Plant Cell 27:1428-1444.

Livak, K. J., and Schmittgen, T. D. 2001. Analysis of relative gene expression data using real-time quantitative PCR and the $2^{-\Delta \Delta C(T)}$ method. Methods 25:402-408.

Luo, J., Butelli, E., Hill, L., Parr, A., Niggeweg, R., Bailey, P., Weisshaar, B., and Martin, C. 2008. AtMYB12 regulates caffeoyl quinic acid and flavonol synthesis in tomato: Expression in fruit results in very high levels of both types of polyphenol. Plant J. 56:316-326.

Luo, J., Xia, W., Cao, P., Xiao, Z., Zhang, Y., Liu, M., Zhan, C., and Wang, N. 2019. Integrated transcriptome analysis reveals plant hormones jasmonic acid and salicylic acid coordinate growth and defense responses upon fungal infection in poplar. Biomolecules 9:12-22.

Major, I. T., Nicole, M. C., Duplessis, S., and Séguin, A. 2010 Photosynthetic and respiratory changes in leaves of poplar elicited by rust infection. Photosynth. Res. 104:41-48. 
Mehrtens, F., Kranz, H., Bednarek, P., and Weisshaar, B. 2005. The Arabidopsis transcription factor MYB12 is a flavonol-specific regulator of phenylpropanoid biosynthesis. Plant Physiol. 138:1083-1096.

Melotto, M., Zhang, L., Oblessuc, P. R., and He, S. Y. 2017. Stomatal defense a decade later. Plant Physiol. 174:561-571.

Mine, A., Seyfferth, C., Kracher, B., Berens, M. L., Becker, D., and Tsuda, K. 2018. The defense phytohormone signaling network enables rapid, high-amplitude transcriptional reprogramming during effector-triggered immunity. Plant Cell 30:1199-1219.

Moffett, P. 2009. Mechanisms of recognition in dominant R gene mediated resistance. Pages 1-229 in: Advances in Virus Research. Elsevier, New York.

Movahedi, S., Van Bel, M., Heyndrickx, K. S., and Vandepoele, K. 2012 Comparative co-expression analysis in plant biology. Plant Cell Environ. 35:1787-1798.

Murase, K., Hirano, Y., Sun, T. P., and Hakoshima, T. 2008. Gibberellininduced DELLA recognition by the gibberellin receptor GID1. Nature 456:459-463.

Mutwil, M., Klie, S., Tohge, T., Giorgi, F. M., Wilkins, O., Campbell, M. M., Fernie, A. R., Usadel, B., Nikoloski, Z., and Persson, S. 2011. PlaNet: Combined sequence and expression comparisons across plant networks derived from seven species. Plant Cell 23:895-910.

Oreiro, E. G., Grimares, E. K., Atienza-Grande, G., Quibod, I. L., RomanReyna, V., and Oliva, R. 2020. Genome-wide associations and transcriptional profiling reveal ROS regulation as one underlying mechanism of sheath blight resistance in rice. Mol. Plant-Microbe Interact. 33:212-222.

Pandey, S. P., Roccaro, M., Schön, M., Logemann, E., and Somssich, I. E. 2010. Transcriptional reprogramming regulated by WRKY18 and WRKY40 facilitates powdery mildew infection of Arabidopsis. Plant J. 64:912-923.

Phillips, P. C. 2008. Epistasis-The essential role of gene interactions in the structure and evolution of genetic systems. Nat. Rev. Genet. 9:855-867.

Pieterse, C. M., Van der Does, D., Zamioudis, C., Leon-Reyes, A., and Van Wees, S. C. 2012. Hormonal modulation of plant immunity. Annu. Rev. Cell Dev. Biol. 28:489-521.

Rajamuthiah, R., and Mylonakis, E. 2014. Effector triggered immunity. Virulence 5:697-702.

Ren, F., Yan, D.-H., Wu, G., Sun, X., Song, X., and Li, R. 2020. Distinctive gene expression profiles and effectors consistent with host specificity in two formae speciales of Marssonina brunnea. Front. Microbiol. 11:276.

Roguev, A., Bandyopadhyay, S., Zofall, M., Zhang, K., Fischer, T., Collins, S. R., Qu, H., Shales, M., Park, H. O., Hayles, J., Hoe, K. L., Kim, D. U., Ideker, T., Grewal, S. I., Weissman, J. S., and Krogan, N. J. 2008. Conservation and rewiring of functional modules revealed by an epistasis map in fission yeast. Science 322:405-410.

Rojas, C. M., Senthil-Kumar, M., Tzin, V., and Mysore, K. S. 2014. Regulation of primary plant metabolism during plant-pathogen interactions and its contribution to plant defense. Front. Plant Sci. 5:17.

Scholes, J. D., and Rolfe, S. A. 1996. Photosynthesis in localised regions of oat leaves infected with crown rust (Puccinia coronata): Quantitative imaging of chlorophyll fluorescence. Planta 199:573-582.

Spiers, A. G. 1984. Comparative studies of host specificity and symptoms exhibited by poplars infected with Marssonina brunnea, Marssonina castagnei and Marssonina populi. For. Pathol. 14:202-218.

Sreekanta, S. 2014. Positive and negative regulation of defense responses against Pseudomonas syringae in Arabidopsis. Ph.D. thesis. University of Minnesota Twin Cities, Minneapolis, MN, U.S.A.

Tameling, W. I. L., and Baulcombe, D. C. 2007. Physical association of the NB-LRR resistance protein $\mathrm{Rx}$ with a Ran GTPase-activating protein is required for extreme resistance to Potato virus X. Plant Cell 19: 1682-1694.

Teixeira, P. J. P. L., Thomazella, D. P., Reis, O., do Prado, P. F., do Rio, M. C., Fiorin, G. L., José, J., Costa, G. G. L., Negri, V. A., Mondego, J. M. C., Mieczkowski, P., and Pereira, G. A. 2014. High-resolution transcript profiling of the atypical biotrophic interaction between Theobroma cacao and the fungal pathogen Moniliophthora perniciosa. Plant Cell 26:4245-4269.

Thimm, O., Bläsing, O., Gibon, Y., Nagel, A., Meyer, S., Krüger, P., Selbig, J., Müller, L. A., Rhee, S. Y., and Stitt, M. 2004. MAPMAN: A user-driven tool to display genomics supplementary data sets onto diagrams of metabolic pathways and other biological processes. Plant J. 37:914-939.
Trapnell, C., Williams, B. A., Pertea, G., Mortazavi, A., Kwan, G., van Baren, M. J., Salzberg, S. L., Wold, B. J., and Pachter, L. 2010. Transcript assembly and quantification by RNA-Seq reveals unannotated transcripts and isoform switching during cell differentiation. Nat. Biotechnol. 28:511-515.

Tufan, H. A., McGrann, G. R. D., Magusin, A., Morel, J. B., Miché, L., and Boyd, L. A. 2009. Wheat blast: Histopathology and transcriptome reprogramming in response to adapted and nonadapted Magnaporthe isolates. New Phytol. 184:473-484.

Tuskan, G. A., Difazio, S., Jansson, S., Bohlmann, J., Grigoriev, I., Hellsten, U., Putnam, N., Ralph, S., Rombauts, S., Salamov, A., Schein, J., Sterck, L., Aerts, A., Bhalerao, R. R., Bhalerao, R. P., Blaudez, D., Boerjan, W. Brun, A., Brunner, A., Busov, V., Campbell, M., Carlson, J., Chalot, M., Chapman, J., Chen, G. L., Cooper, D., Coutinho, P. M., Couturier, J., Covert, S., Cronk, Q., Cunningham, R., Davis, J., Degroeve, S., Déjardin, A., Depamphilis, C., Detter, J., Dirks, B., Dubchak, I., Duplessis, S., Ehlting, J., Ellis, B., Gendler, K., Goodstein, D., Gribskov, M., Grimwood, J., Groover, A., Gunter, L., Hamberger, B., Heinze, B., Helariutta, Y., Henrissat, B., Holligan, D., Holt, R., Huang, W., IslamFaridi, N., Jones, S., Jones-Rhoades, M., Jorgensen, R., Joshi, C., Kangasjärvi, J., Karlsson, J., Kelleher, C., Kirkpatrick, R., Kirst, M. Kohler, A., Kalluri, U., Larimer, F., Leebens-Mack, J., Leplé, J. C., Locascio, P., Lou, Y., Lucas, S., Martin, F., Montanini, B., Napoli, C., Nelson, D. R., Nelson, C., Nieminen, K., Nilsson, O., Pereda, V., Peter, G., Philippe, R., Pilate, G., Poliakov, A., Razumovskaya, J., Richardson, P., Rinaldi, C., Ritland, K., Rouzé, P., Ryaboy, D., Schmutz, J., Schrader, J., Segerman, B., Shin, H., Siddiqui, A., Sterky, F., Terry, A., Tsai, C. J., Uberbacher, E., Unneberg, P., Vahala, J., Wall, K., Wessler, S., Yang, G., Yin, T., Douglas, C., Marra, M., Sandberg, G., Van de Peer, Y., and Rokhsar, D. 2006. The genome of black cottonwood, Populus trichocarpa (Torr. \& Gray). Science 313:1596-1604.

van Loon, L. C. 1985. Pathogenesis-related proteins. Plant Mol. Biol. 4: 111-116.

van Loon, L. C., Rep, M., and Pieterse, C. M. J. 2006. Significance of inducible defense-related proteins in infected plants. Annu. Rev. Phytopathol. 44:135-162.

Wang, L., Wang, B., Du, Q., Chen, J., Tian, J., Yang, X., and Zhang, D. 2017. Allelic variation in PtoPsbW associated with photosynthesis, growth, and wood properties in Populus tomentosa. Mol. Genet. Genomics 292:77-91.

Weaver, L. M., Swiderski, M. R., Li, Y., and Jones, J. D. G. 2006. The Arabidopsis thaliana TIR-NB-LRR R-protein, RPP1A; protein localization and constitutive activation of defence by truncated alleles in tobacco and Arabidopsis. Plant J. 47:829-840.

Whitham, T. G., Difazio, S. P., Schweitzer, J. A., Shuster, S. M., Allan, G. J., Bailey, J. K., and Woolbright, S. A. 2008. Extending genomics to natural communities and ecosystems. Science 320:492-495.

Yanhui, C., Xiaoyuan, Y., Kun, H., Meihua, L., Jigang, L., Zhaofeng, G., Zhiqiang, L., Yunfei, Z., Xiaoxiao, W., Xiaoming, Q., Yunping, S., Li, Z., Xiaohui, D., Jingchu, L., Xing-Wang, D., Zhangliang, C., Hongya, G., and Li-Jia, Q. 2006. The MYB transcription factor superfamily of Arabidopsis: Expression analysis and phylogenetic comparison with the rice MYB family. Plant Mol. Biol. 60:107-124.

Zhang, Y., He, W., and Yan, D.-H. 2018a. Histopathologic characterization of the process of Marssonina brunnea infection in poplar leaves. Can. J. For. Res. 48:1302-1310.

Zhang, Y., He, W., Yan, D. H., and Fossdal, C. G. 2018b. Genome-wide identification and development of microsatellite markers for Marssonina brunnea and their applications in two populations. For. Pathol. 48: e12433.

Zhang, Y., Tian, L., Yan, D.-H., and He, W. 2018c. Genome-wide transcriptome analysis reveals the comprehensive response of two susceptible poplar sections to Marssonina brunnea infection. Genes (Basel) 9:154.

Zhang, Y., Zhang, X., Chen, Y., Wang, Q., Wang, M., and Huang, M. 2007. Function and chromosomal localization of differentially expressed genes induced by Marssonina brunnea f. sp. multigermtubi in Populus deltoides. J. Genet. Genomics 34:641-648.

Zhu, S., Dai, Y. M., Zhang, X. Y., Ye, J. R., Wang, M. X., and Huang, M. R. 2013. Untangling the transcriptome from fungus-infected plant tissues. Gene 519:238-244. 